\title{
The role of Colk7 in CAK function, a retro-retrospective
}

\author{
J. Wade Harper ${ }^{1,4}$ and Stephen J. Elledge ${ }^{1-4}$ \\ ${ }^{1}$ Verna and M arrs M cLean Department of Biochemistry and ${ }^{2}$ Department of M olecular and Human Genetics, ${ }^{3} \mathrm{Howard}$ \\ Hughes Medical Institute, Baylor College of Medicine, Houston, Texas, 77030 USA
}

Most transitions in the eukaryotic cell cycle are catalyzed by cyclin-dependent kinases (Cdks, CDKs), complexes formed between a member of the Cdk family of protein kinases and a regulatory cyclin subunit. CDKs are among the most highly regulated enzymes known: Their activities are controlled through multiple mechanisms that include cyclin association, positive and negative phosphorylation events, negative regulation through association with Cdk inhibitors (CKIs), and association with accessory proteins such as Cks/Sucl (Fig. 1) (reviewed in Morgan 1997). In addition to controlling C dk activation, the cyclin subunit may also contribute to substrate specificity. These elaborate regulatory pathways reflect the critical roles of cyclin-kinases in the life of a cell; alterations that generate unregulated cyclinkinase activity can promote improper proliferation that can result in developmental defects or proliferative diseases such as cancer (Sherr 1996).

The Cdk subunit alone is inactive and requires both association with a cyclin and phosphorylation on a conserved threonine residue (T 161 in human C dc2 and T 160 in human Cdk2) for full activation (Gould et al. 1991; Desai et al. 1992; Solomon et al. 1992; Connell-Crowley et al. 1993). In the case of Cdk2, these two events account for an increase in activity of greater than seven orders of magnitude (Connell-C rowley et al. 1993; Russo 1997). Cyclin A promotes Cdk2 activation by inducing two major structural changes in the kinase that are likely to be conserved in other cyclin/C dk pairs (Jeffrey et al. 1996; Russo et al . 1996). First, cyclin binding leads to a conformational change in the amino-terminal PSTAIRE/helix that reorients the catalytic residue glutamate-51. This residue is conserved in all eukaryotic protein kinases and is involved in ATP binding. In addition, cyclin binding leads to a change in the orientation of the T-loop, the region of $\mathrm{Cdk} 2$ containing the activating threonine phosphorylation site T160. In the absence of cyclin, the T-loop blocks substrate access to the active site but the conformational changes induced by cyclin largely relieve this steric block. This conformational change in the T-loop may al so be critical for the activating phosphorylation event because T160 is not particu-

4E-MAIL jharper@bcm.tmc.edu; selledge@bcm.tmc.edu; FAX (713) 7969438. larly solvent-exposed in monomeric Cdk2 but is well exposed in the cyclin/Cdk2 complex. Completion of the $\mathrm{Cdk}$ activation process involves phosphorylation of the $\mathrm{T}$-loop threonine by Cdk activating kinase (CAK), a modification that promotes further structural changes in the T-loop (Russo et al. 1996).

\section{Evidence for Colk7/cyclin H as CAK}

There has been an overwhelming interest in the elucidation of $\mathrm{Cdk}$ regulatory mechanisms since they ultimately control cell cycle transitions and, therefore, proliferations. This was particularly true for CAK since blocking this activation step would provide an effective means of inhibiting the activity of perhaps all C dk complexes. The first indications of the existence of CAK came from the identification of an activity in Xenopus egg (Solomon et al. 1992) and mammalian cell extracts (Desai et al. 1992) that would activate Cdc2 through phosphorylation of T161, a process that requires prior association with cyclin B. Using Cdk activation as an assay, a CAK activity was purified from multiple systems and the activity was attributed to a complex between C dk 7 (originally referred to as MO15 in Xenopus) (Fesquet et al. 1993; Poon et al. 1993; Solomon et al. 1993) and cyclin H (Fisher and Morgan 1994; Mäkelä et al. 1994). This complex was subsequently shown to activate cyclin D/C dk4 complexes (Matsuoka et al. 1994) and homologs in S. pombe were identified that displayed CAK activity in vitro (Buck et al. 1994; Damagnez et al. 1995). There was good reason to believe that this CAK activity constituted the major CAK in Xenopus and mammalian cells. First, there was no biochemical evidence for additional CAK activities distinct from those isolated, and their activity tracked as a single peak over several purification steps (Solomon et al. 1993; Fisher and Morgan 1994). Consistent with this, antibodies against $\mathrm{XeC} d \mathrm{k} 7$ depl eted CAK activity from egg extracts and $\mathrm{XeCdk} 7$ immune complexes contain CAK activity that phosphorylates Cdk2 specifically on T 160 (Poon et al. 1993; Solomon et al. 1993; Fesquet et al. 1997). Second, recombinant $\mathrm{Cdk} 7$ and cyclin $\mathrm{H}$ al one can reconstitute CAK activity toward Cdc2 and Cdk2 (Fisher and Morgan 1994), providing strong evidence that the activity attributed to these proteins in purified fractions 


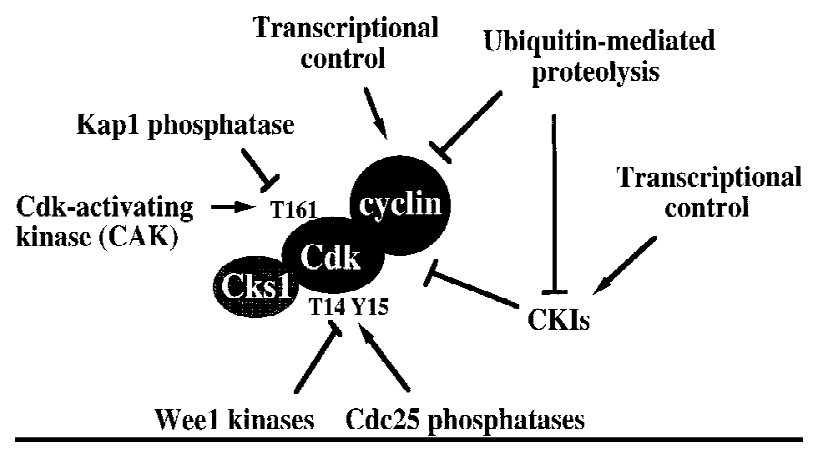

Figure 1. Multiple mechanisms regulate Cdk activity. Cdk activity is positively regulated (arrowheads) by association with cyclins, by phosphorylation on T161 by CAK, and by dephosphorylation by $\mathrm{Cdc} 25$ phosphatases. Cdk activity is negatively regulated by CKIs, Weel-like kinases, and possibly by Kapl phosphatase. The abundance of cyclins and some CKIs is regulated by transcriptional mechanisms and by ubiquitin-mediated proteolysis. The function of Cks1 is unknown but may involve substrate recognition.

resulted from the $\mathrm{Cdk} 7 /$ cyclin $\mathrm{H}$ complex. In contrast to expectations, Cdk7/cyclin $\mathrm{H}$ activity appears to be constitutive during the cell cycle (Poon et al. 1994; Tassan et al. 1994), suggesting that CAK is not used specifically to regulate specific cell cycle transitions, at least in a particular cell type under the conditions examined. N evertheless, these findings were intriguing in that they demonstrated the involvement of a C dk-based ki nase cascade in cyclin-kinase activation.

$\mathrm{Cdk} 7 /$ cyclin $\mathrm{H}$ is unusual in that its activation can proceed through two distinct pathways. One pathway involves enhanced association of $\mathrm{Cdk} 7$ and cyclin $\mathrm{H}$ through an assembly factor referred to as p36 $6^{\text {MATI }}$ (Devault et al. 1995; Fisher et al. 1995; Tassan et al. 1995). In this case, MAT 1 is a stable component of the CAK complex and activation proceeds without a requirement for phosphorylation of $\mathrm{Cdk} 7$ in its T-loop equivalent. MATI contains a RING finger domain, although assembly activity does not require this domain. An alternative pathway for activation requires phosphorylation of $\mathrm{Cdk} 7$ in its T-loop and this function can be provided by cyclin A/Cdk2 in vitro, suggesting a possible feedback loop in Cdk activation (Fisher et al. 1995).

\section{Complications arise concerning the role of $\mathrm{Cdk} 7 /$ cyclin $\mathrm{H}$ as CAK}

The early biochemical data supporting a role for $\mathrm{Cdk} 7 /$ cyclin $\mathrm{H}$ in $\mathrm{Cdk}$ activation was strong. However, this simple picture of $\mathrm{Cdk} 7 /$ cyclin $\mathrm{H}$ function was complicated by genetic and biochemical data demonstrating that the $\mathrm{Cdk} 7$ homolog in Saccharomyces cerevisiae, $\mathrm{Kin28}$, has a role in transcription (reviewed in Fisher and Morgan 1996). Both the mammalian Cdk7/cyclin H/ MAT1 complex and its most closely related budding yeast complex, Kin28/Ccl1 (Valay et al. 1993), were shown to be components of the basal transcription com- plex TFIIH (Feaver et al. 1994; Roy et al. 1994; Serizawa et al. 1995; Shiekhattar et al. 1995; Valay et al. 1995; Svejstrup et al. 1996). TFIIH plays critical roles in both transcription initiation and in nucleotide excision repair and was known for some time to contain an activity capable of phosphorylating the RNA polymerase II carboxy-terminal domain (CTD). Thus the finding that a Cdk complex is a component of TFIIH immediately suggested that this kinase might contribute to CTD phosphorylation. Perhaps not surprisingly, Cdk7 activity in TFIIH complexes is required for transcription of some (but not all) promoters in vitro (Akoulitchev et al. 1995; Mäkelä et al. 1995) and TFIIH-associated Cdk7 activity toward the CTD does not appear to be required for the initiation step in vitro (Akoulitchev et al. 1995). Interestingly, cyclin/Cdk complexes are used in multiple contexts in transcription. For example, Cdk8/cyclin C is a component of the RNA polymerase holoenzyme and also acts as a CTD kinase in vitro (Liao et al. 1995; Leclerc et al. 1996; Rickert et al. 1996). Importantly, both free and TFIIH-associated Cdk7/cyclin H/MAT 1 complexes exist in cell extracts, and the free C $\mathrm{dk} 7$ complex has a higher specific CAK activity than the TFIIH complex (Drapkin et al. 1996; Rossignol et al. 1997; YankuIov and Bentley 1997). N evertheless, the appearance of $\mathrm{Cdk} 7 / \mathrm{cyclin} \mathrm{H}$ as a component of TFIIH was sufficient to raise some doubt as to whether this kinase functioned as CAK in vivo.

Evidence against cyclin $\mathrm{H} / \mathrm{Cdk} 7$ complexes acting as CAK in vivo

Although the suggestion that $\mathrm{Cdk} 7 / \mathrm{cyclin} \mathrm{H}$ might function in transcription did not rule out a role in direct $\mathrm{Cdk}$ regulation, it did raise the question of whether it really had two functions in vivo. Doubt concerning the role of the cyclin $\mathrm{H} / \mathrm{Cdk} 7$ complex as a CAK in vivo was further compounded by genetic and biochemical data demonstrating that $\mathrm{Kin} 28$ has a role in transcription and is a CTD kinase, but is not required for $\mathrm{Cdc} 28$ phosphorylation in vivo and cannot act as CAK in vitro (Cismowski et al. 1995).

These findings led to a search for the actual in vivo CAK function in yeast. The answer came last year with the identification of a novel kinase Cak1 (al so referred to as Civ1 for CAK in vivo) (Espinoza et al. 1996; Kaldis et al. 1996; Thuret et al. 1996). Although Cak1 was also identified based on its ability to phosphorylate Cdc28 and Cdk2 in an in vitro assay (Espinoza et al. 1996; Kaldis et al. 1996), mutational analysis of the CAK1 gene provided an in vivo verification that Cak 1 is required for Cdc28 phosphorylation and activation. Temperaturesensitive mutations were identified that arrest at either the G1/S transition or the G2/M transition (Kaldis et al. 1996; Thuret et al. 1996), the two transitions that are most sensitive to defects in Cdc28 activation. Unlike $\mathrm{Cdk} 7 /$ cyclin $\mathrm{H}, \mathrm{Cak} 1$ is active as a monomer and lacks CTD kinase activity. Although it is most closely related to the Cdk family of protein kinases, the kinase is un- 
usual, in that it lacks 4 of the 12 signature motifs in protein kinases.

\section{The return of $\mathrm{Cdk} 7$ as CAK}

There are two simple explanations for the discrepancy concerning the identity of CAK. The first is that metazoans contain a CAK1 homolog that is responsible for Cdk activation, and that $\mathrm{Cdk} 7 /$ cyclin $\mathrm{H} / \mathrm{MAT} 1$ functions primarily in the context of TFIIH but has the ability to phosphorylate Cdks in vitro. The second explanation is that budding yeast utilize a distinct mechanism for Cdk activation and that Cdk7 is actually a bifunctional enzyme in larger eukaryotes, promoting both $\mathrm{C} \mathrm{dk}$ activation and transcription. At first glance, the possi bility of a distinct mechanism in budding yeast seems unlikely. Most fundamental cell cycle control mechanisms are conserved throughout all eukaryotes. However, a recent genetic analysis of $\mathrm{Cdk} 7$ function suggests that the string of conserved cell cycle regulators may have just been broken with respect to CAK activity. An article in this issue of Genes \& Development provides strong genetic evidence for a role of $\mathrm{Cdk} 7$ in $\mathrm{Cdc} 2$ activation in Drosophila melanogaster (Larochelle et al. 1997). Although the results in this paper do not rule out the existence of a CA K1-rel ated gene in species other than budding yeast, they do promote the notion that one of the most fundamental aspects of Cdk activation occurs through distinct mechanisms in budding yeast and at least some metazoans.

A ntibodies against DmCdk7 immunoprecipitate CAK activity toward $\mathrm{Cdk} 2 / \mathrm{cyclin}$ A from embryo extracts. Most importantly, cdk $7^{\text {null }}$ animals display defects in progression through the larval stage: Approximately half of the mutant animals die as larvae whereas the remainder die as pupae. Defects in cell proliferation are most pronounced in the imaginal discs where mitotic activity is high in the devel oping embryo. In contrast, there is no apparent defect in polyploid tissues where cells undergo multiple $\mathrm{S}$ phases without mitosis, a process that is thought to require cyclin E/Cdk2 (a.k.a. cdc2c). Consistent with a role in $\mathrm{Cdc} 2$ activation, these phenotypes closely parallel those seen in Dmcdc2 mutants (Stern et al. 1993).

The effects on imaginal disc development in $\mathrm{cdk} 7^{\text {null }}$ embryos, although consistent with a requirement for cdk7 in cell proliferation, could be indi rect. More definitive experiments came from the analysis of a conditional Cdk7 allele. cdk7ts animals were shown to be viable at $18^{\circ} \mathrm{C}$ but inviable at $27^{\circ} \mathrm{C}$ (Larochelle et al. 1997). The mutant Cdk7 protein was not temperature-sensitive for CAK activity in vitro, but did show a progressive reduction in cdk7 abundance over time in vivo. This complicates the analysis somewhat as the low levels of C $\mathrm{dk} 7$ that persist may retain some activity. When shifted to restrictive temperatures, adult animals remain viable over an extended period of time but display defects in gamete production, the timing of which correlates with reduced cdk7 protein. Similar gametogenesis defects were detected in cdc2 mutants.
The analysis of Dmcdk7 mutations is complicated by the possibility that this kinase is required not only for Cdk phosphorylation but may also be required for expression of proteins required for cell cycle progression, including cyclins, through its association with TFIIH. For example, in budding yeast, inactivation of Kin28 leads to reduced levels of Cdc28-dependent kinase activity not because of defects in Cdc28 phosphorylation but because of defects in cyclin gene transcription (Cismowsky et al. 1995). This issue was addressed in part by demonstrating that the total levels of A-, B-, and E-type cyclins remain high in cdk7ts animals (Larochelle et al. 1997).

The availability of cdk7ts al leles al so provided an opportunity to examine the status of cyclin/Cdk complexes and activities in mutant animals. In contrast to mammals, Drosophila cyclins A and B form exclusive complexes with $\mathrm{Cdc} 2$ whereas cyclin $\mathrm{E}$ specifically interacts with Cdk2. Loss of cdk7 function leads to decreased cyclin A-dependent kinase activity largely through dissociation of $\mathrm{Cdc} 2$ whereas decreased cyclin B-dependent activity correl ates with decreased levels of T161-phosphorylated Cdc2. Reduced levels of Cdc2 phosphorylation in cdk7ts mutants provides the strongest data to date linking $\mathrm{Cdk} 7$ function to $\mathrm{Cdk}$ activation. The results with cyclin A are in agreement with previous biochemical studies indicating a role for T161 phosphorylation in stabilization of cycl in A / C dc2 complexes (Ducommun et al. 1991; Desai et al . 1995). Surprisingly, however, cyclin E-dependent kinase levels were not affected in the cdk7ts mutant (Larochelle et al. 1997), consistent with the unimpaired endoreduplication of the null allele.

If C dk 7 controls $\mathrm{Cdc} 2$ activation in Drosophila, does it also have a role in transcription? One prediction would be that if $\mathrm{Cdk} 7$ plays a major role in transcription, its loss might have substantial effects on gene expression and affect many pathways, as Kin28 loss appears to do. In this regard, the ability to undergo multiple S-phases was used as an indirect argument for the absence of global effects on transcription with loss of cdk7 function (Larochelle et al. 1997). However, given the fact that residual wild-type $\mathrm{Cdk} 7$ activity might remain in the mutants either from maternal stores or from incomplete inactivation of the ts allele, further studies are required to determine to what extent, if any, TFIIH function is altered in cdk 7 mutant animal s. It should be noted that, in the absence of complete genome information, a C dk7related gene could carry out the transcriptional role.

\section{CAK questions}

The picture of CAK now emerging is that C dk7 is likely to be a bifunctional protein in metazoans but that two proteins, Kin28 and Cak1, fulfill these functions in budding yeast. However this picture is only a rough sketch with many important details yet to be added. Is CAK really an integral component of the transcriptional apparatus? Is it regulated, and if so how? Will there be additional Cdk7- and Cak1-related molecules that differen- 
tially regulate $\mathrm{Cdks}$ and transcription during development? And precisely what is the relationship between Cdk7 and the different Cdk/cyclin complexes in cells? If $\mathrm{Cdk} 7 /$ cyclin $\mathrm{H}$ is truly CAK, does it regulate cyclin $\mathrm{E} /$ Cdk2 activity in Drosophila? It is conceivable that C dk2 regulation in Drosophila occurs through a distinct CAK, perhaps Cak1-related kinase (oh, the agony of an incomplete genome project). Alternatively, the answer may lie not in the CAK phosphorylation step itself but in its reversal, T160-dephosphorylation by the Kap1 phosphatase (Poon and Hunter 1995). This phosphatase binds and dephosphorylates $\mathrm{Cdk} 2$ and $\mathrm{Cdc} 2$, but only in the absence of cyclin, that is, after the cyclin subunit has been destroyed through ubi quitin-mediated proteolysis. Thus, the persistence of cyclin E-dependent kinase activity may reflect an inability to destroy the existing cyclin $\mathrm{E}$, either because its destruction machinery is not active in G2, the likely arrest point, or possibly because its destruction requires cyclin A activity, which is absent in cdk7 mutant cells. In the presence of cyclin E, Cdk2 would be inaccessible to Kapl and maintain its activity despite the absence of $\mathrm{Cdk} 7$ activity. A trivial explanation for the results with cyclin $E$ that also cannot be excluded is that $\mathrm{Cdk} 7$ has a higher specific activity for cyclin $\mathrm{E} / \mathrm{Cdk} 2$ and that residual Cdk7 activity present at the nonpermissive temperature preferentially maintains $\mathrm{Cdk} 2$ in its activated state relative to $\mathrm{Cdc} 2$. Thus, it is concei vable that $\mathrm{Cdk} 7$ is the CAK for cyclin E/Cdk2 but the in vivo conditions were unable to reveal this. Only further studies will distinguish between these possibilities.

In arriving at a complete understanding of CAK biology, one must wrestle with the issue of why Cdk function evolved to require CAK activation in the first place. The most satisfying answer would be that CAK activity is regulated, but evidence supporting this explanation has been elusive. Although the CAK itself may not be regulated, its access to Cdks could be manipulated by Cdk binding proteins as has been suggested for the CKI p27 (Kato et al. 1994). Because biochemical purification might obscure actual CAK regulation (the biochemical equivalent of the Heisenberg uncertainty principle), in vivo assays for CAK activity that do not depend on C dks may need to be developed to explore in vivo CAK regulation. On the other hand, if CAK activity is simply constitutive and dictated by the presence of cyclin, then why did cyclins and Cdks not evolve the ability to allow cyclins to function fully in Cdk activation, leaving negative regulation to tyrosine phosphorylation and Cdk inhibitors? One possibility is that the effect of phosphothreonine on Cdk structure cannot be simply mimicked by any naturally occurring amino acid, which is al so suggested by the finding that negatively charged amino acids will not substitute for phosphothreonine in Cdk activation (Gould et al. 1991; Connell-Crowley et al. 1993).

Although these breakthroughs have certainly clarified the role of $\mathrm{Cdk} 7$ considerably, this defined break in the conservation of basic cell cycle regulators among eukaryotes is enough to send ripples of concern through any argument based on evolutionary conservation. In this light, it should be noted that, al though we now have strong evidence that $\mathrm{Cdk} 7$ is behaving as CAK in Drosophila, this does not necessarily mean that the same will hold true for mammals. However, for the time being we have reason to bel ieve that $\mathrm{Cdk} 7$ has a good chance to be involved in $\mathrm{Cdk}$ regulation in all metazoans. Of course, we will just have to see how long this revised notion of cell cycle conservation holds.

\section{Acknowledgments}

We thank Mark Solomon and David M organ for hel pful discussions and apologize to those whose work could not be cited directly because of space limitations. Research in the authors' laboratories is supported by $\mathrm{N}$ ational Institutes of Heal th grants GM 44664 to S.J.E. and GM 54137 to J.W.H., and by the Welch Foundation.

\section{References}

Akoulitchev, S., T.P. M äkelä, R.A. Weinberg, and D. Reinberg. 1995. Requirement for TFIIH kinase activity in transcription by RNA polymerase II. Nature 377: 557-560.

Buck, V., P. Russell, and J.B.A. Millar. 1995. Identification of a cdk-activating kinase in fission yeast. EMBO J. 14: 61736183.

Cismowsky, M.J., G.M. Laff, M.J. Solomon, and S.I. Reed. 1995. Kin28 encodes a C-terminal domain kinase that controls mRN A transcription in Saccharomyces cerevisiae but lacks Cyclin-dependent kinase-activating kinase (CAK) activity. Mol. Cell. Biol. 15: 2983-2992.

Connell-Crowley, L., M.J. Solomon, N. Wei, and J.W. Harper. 1993. Phosphorylation-independent Activation of human cyclin-dependent kinase 2 by cyclin A in vitro. Mol. Biol. Cell 4: 79-92.

Damagnez, V., T.P. Mäkelä, and G. Cottarel. 1995. Schizosaccharomyces pombe Mop1-Mcs2 is related to mammalian CAK. EMBO J. 14: 6164-6172.

Desai, D., Y. Gu, and D.O. M organ. 1992. Activation of human cyclin-dependent kinases in vitro. Mol. Biol. Cell 3: 571582.

Desai, D., H.C. Wessling, R.P. Fisher, and D.O. Morgan. 1995. Effects of phosphorylation by CAK on cyclin binding by CDC2 and CDK2. Mol. Cell. Biol. 15: 345-350.

Devault, A., A.-M. Martinez, D. Fesquet, J.-C. Labbé, N. M orin, J.-P. Tassan, E.A. Nigg, J.-C Cavadore, and M. Doree. 1995. MAT1 ("ménage á trois") a new RIN G finger protein subunit stabilizing cyclin $\mathrm{H}$-cdk 7 complexes in starfish and Xenopus CAK. EMBO J. 14: 5027-5036.

Drapkin, R., G. Le Roy, H. Cho, S. Akoulitchev, and D. Reinberg. 1996. Human cyclin-dependent kinase-activating kinases exist in three distinct complexes. Proc. Natl. Acad. Sci. 93: 6488-6493.

Ducommun, B., P. Brambilla, M.A. Felix, B.R. Franza Jr., E. Karsenti, and G. Draetta. 1991. cdc2 phosphorylation is required for its interaction with cyclin. EMBO J. 10: 33113319.

Espinoza, F.H., A. Farell, H. Erdjument-Bromage, P. Tempts, and D.O. Morgan. 1996. A Cyclin-dependent kinase-activating kinase (CAK) in budding yeast unrelated to vertebrate CAK. Science 273: 1714-1717.

Feaver, W.J., J.Q. Svejsrup, N.L. Henry, and R.D. Kornberg. 1994. Relationship of CDK-activating kinase and RNA polymerase II CTD kinase TFIIH/TFIIK. Cell 79: 1103-1109. 
Fesquet, D., N. Morin, M. Dorée, and A. Devault. 1997. Is Cdk7/cyclin H/MAT1 the genuine cdk activating kinase in cyclin Xenopus egg extracts? Oncogene 15: 1303-1307.

Fesquet, D., J.-C. Labbé, J. Derancourt, J.-C. Capony, S. Gal as, F. Girard, T. Lorca, J. Shuttleworth, M. Dorée, and J.-C. Cavadore. 1993. The M 015 gene encodes the catalytic subunit for a protein kinase that activates cdc2 and other cyclin-dependent kinases (CDKs) through phosphorylation of Thr161 and its homologues. EMBO J. 12: 3111-3121.

Fisher, R.P. and D.O. Morgan. 1994. A novel form of cyclin associates with MO15/CDK7 to form the CDK-activating kinase. Cell 78: 713-724.

- - 1996. CAK in TFIIH: Crucial connection or confounding coincidence? Biochim. Biophys. Acta 1288: 7-10.

Fisher, R.P., P. Jin, H.M. Chamberlin, and D.O. Morgan. 1995. Alternative mechanisms of CAK assembly require an assembly factor or an activating kinase. Cell 83: 47-57.

Gould, K.L., S. M oreno, D.J. Owen, S. Sazer, and P. N urse. 1991. Phosphorylation at Thr167 is required for Schizosaccharomyces pombe p34cdc2 function. EMBO J. 10: 3297-3309.

Jeffrey, P.D., A.A. Russo, K. Polyak, E. Gibbs, J. Hurwitz, J. Massagué, and N.P. Pavletich. 1995. Mechanism of CDK activation by the structure of a cyclin A-CDK2 complex. Nature 376: 313-320.

Kaldis, P., A. Sutton, and M.J. Solomon. 1996. The Cdk-activating kinase (CAK) from budding yeast. Cell 86: 553-564.

Kato, J.Y., M. Matsuoka, K. Polyak, J. Massagué, and C.J. Sherr. 1994. Cyclic AMP-induced G1 phase arrest mediated by an inhibitor (p27Kip1) of cyclin-dependent kinase 4 activation. Cell 79: 487-496

Larochelle, S., J. Pandur, R.P. Fisher, H.K. Salz, and B. Suter. 1997. Cdk7 is essential for mitosis and for in vivo Cdk-activating kinase (CAK) activity. Genes \& Dev., (this issue).

Leclerc, V., J.P. Tassan, P.H. O'Farrell, E.A. Nigg, and P. Leopold. 1996. Drosophila Cdk8, a kinase partner of cyclin C that interacts with the large subunit of RN A polymerase II. Mol. Biol. Cell 7: 505-513.

Liao, S.M., J. Zhang, D.A. Jeffery, A.J. Koleske, C.M. Thompson, D.M. Chao, M. Viljoen, H.J. van Vuuren, and R.A. Young. 1995. A kinase-cyclin pair in the RNA polymerase II holoenzyme. Nature 374: 193-196.

Mäkelä, T.P., J.D. Parvin, J. Kim, J.L. Huber, P.A. Sharp, and R.A. Weinberg. 1995. A kinase-deficient transcription factor TFIIH is functional in basal and activated transcription. Proc. Natl. Acad. Sci. 92: 5174-5178.

Mäkelä, T.P., J.P. Tassan, E.A. N igg, S. Frutiger, G.J. Hughes, and R.A. Weinberg. 1994. A cyclin associates with the CDKactivating kinase M O15. Nature 371: 254-257.

Matsuoka, M., J.-Y. Kato, R.P. Fisher, D.O. Morgan, and C.S. Sherr. 1994. Activation of cyclin-dependent kinase 4 (cdk4) by mouse M O15-associated kinase. Mol. Cell Biol. 14: 72657275.

Morgan, D.O. 1997. Cyclin-dependent kinases: Engines, clocks and microprocessors. Annu. Rev. Cell Dev. Biol. 13, (in press).

Poon, R.Y.C. and T. Hunter. 1995. Dephosphorylation of Cdk2 Thr160 by the cyclin-dependent kinase-interacting phosphatase KAP in the absence of cyclin. Science 270: 90-93.

Poon, R.Y.C., K. Yamashita, J.P. Adamczewszi, T. Hunt, and J. Shuttleworth. 1993. The cdc2-related protein $\mathrm{p} 40^{\mathrm{MO} O 15}$ is the catalytic subunit of a protein kinase that can activate $\mathrm{p} 33^{\mathrm{cdk} 2}$ and p34 ${ }^{\mathrm{cdc}}$. EMBO J. 12: 3123-3132.

Poon, R.Y.C., K. Yamashita, M. Howell, M.A. Ershier, A. Belyavsky, and T. Hunt. 1994. Cell cycle regulation of the p34 ${ }^{\mathrm{cdc2}} / \mathrm{p} 33^{\mathrm{cdk} 2}$-activating kinase $\mathrm{p} 40^{\mathrm{MO} 15}$. J. Cell Sci. 107: 2789-2799.
Rickert, P., W. Seghezzi, F. Shanahan, H. Cho, and E. Lees. 1996. Cyclin C/CDK8 is a novel CTD kinase associated with RN A polymerase II. Oncogene 12: 2631-2640.

Rossignol, M., I. Kolb-Cheynel, and J.M. Egly. 1997. Substrate specificity of the cdk-activating kinase (CAK) is al tered upon association with TFIIH. EMBO J. 16: 1628-1637.

Roy, R., J.P. Adamczewszi, T. Seroz, W. Vermeulen, J.-P. Tassan, L. Schaeffer, E.A. Nigg, J.H.J. Hoeijmakers, and J.-M . Egly. 1994. The MO15 cell cycle kinase is associated with the TFIIH transcription-DNA repair factor. Cell 79: 10931101.

Russo, A.A. 1997. Purification and reconstitution of cyclin-dependent kinase 2 in four states of activity. Meth. Enzymol. 283: 3-12.

Russo, A.A., P.D. Jeffrey, and N.P. Pavlevitch. 1996. Structural basis of cyclin-dependent kinase activation by phosphorylation. Nature Str. Biol. 3: 696-700.

Serizawa, H., T.P. M äkelä, J.W. Conaway, R.C. Canaway, R.A. Weinberg, and R.A. Young. 1995. Association of cdk-activating kinase subunits with transcription factor TFIIH. Nature 374: 280-282.

Sherr, C.J. 1996. Cancer cell cycles. Science 274: 1672-1677.

Shiekhattar, R., F. Mermelstein, R.P. Fisher, R. Drapkin, B. Dynlacht, H.C. Wessling, D.O. Morgan, and D. Reinberg. 1995. Cdk-activating kinase complex is a component of human transcription factor TFIIH. Nature 374: 283-287.

Solomon, M.J., T. Lee, and M.W. Kirschner. 1992. The role of phosphorylation in p34 cdc2 activation: Identification of an activating kinase. Mol. Biol. Cell 3: 13-27.

Solomon, M.J., J.W. Harper, and J. Shuttleworth. 1993. CAK, the p34 ${ }^{\text {cdc2 }}$ activating kinase, contains a protein identical or closely related to $440^{\mathrm{MO}}{ }^{15}$. EMBO J. 12: 3133-3142.

Stern, B., G. Ried, N.J. Clegg, T.A. Grigliatti, and C.F. Lehner. 1993. Genetic analysis of the Drosophila cdc2 homolog. Development 117: 219-232.

Svejstrup, J.Q., W.J. Feaver, and R.D. Kornberg. 1996. Subunits of RN A polymerase II transcription factor TFIIH encoded by the CCL1 gene. J. Biol. Chem. 271: 643-645.

Tassan, J.-P., S.J. Schultz, J. Bartek, and E.A. Nigg. 1994. Cell cycle analysis of the activity, subcellular localization, and subunit composition of human CAK (CDK-activating Kinase). J. Cell Biol. 127: 467-478.

Tassan, J.-P., M. Jaquenoud, A.M. Fry, S. Frutiger, G.J. Hughes, and E.A. N igg. 1995. In vitro assembly of a functional human CDK7-cyclin $\mathrm{H}$ complex requires MAT1, a novel $36 \mathrm{kDa}$ RING finger protein. EMBO J. 14: 5608-5617.

Thuret, J.-Y., J.-G. Valay, G. Faye, and C. Mann. 1996. Civ1 (CAK in vivo), a novel Cdk-Activating Kinase. Cell 86: 565576.

Valay, J.G., M. Simon, and G. Faye. 1993. The Kin28 protein kinase is associated with a cyclin in Saccharomyces cerevisiae. J. Mol. Biol. 234: 307-310.

Valay, J.G., M. Simon, M.F. Dubois, O. Bensaude, C. Faca, and G. Faye. 1995. The KIN 28 gene is required both for RNA polymerase II mediated transcription and phosphorylation of the Rpb1p CTD. J. Mol. Biol. 234: 307-310.

Yankulov, K.Y. and D.L. Bentley. 1997. Regulation of CDK7 substrate specificity by MAT1 and TFIIH. EMBO J. 16: 1638-1646. 


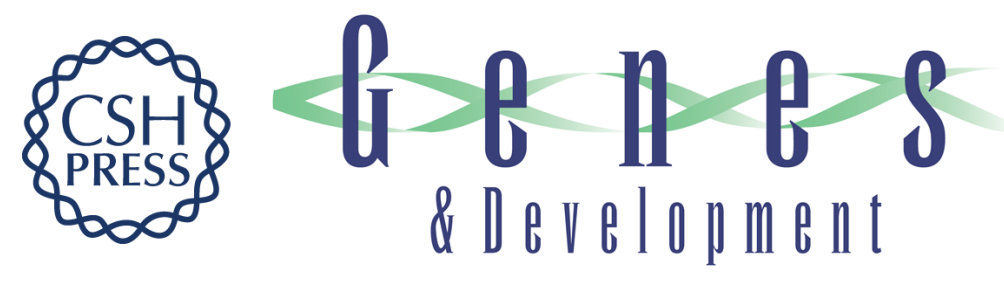

\section{The role of Cdk7 in CAK function, a retro-retrospective}

J. Wade Harper and Stephen J. Elledge

Genes Dev. 1998, 12:

References This article cites 46 articles, 18 of which can be accessed free at: http://genesdev.cshlp.org/content/12/3/285.full.htmI\#ref-list-1

License

Email Alerting

Receive free email alerts when new articles cite this article - sign up in the box at the top Service right corner of the article or click here.

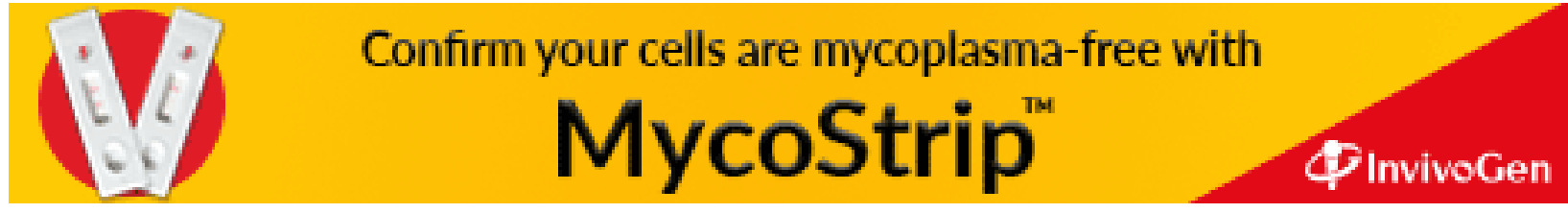

